\title{
Morphological Variability of Neglected and Underutilized Legumes (NULs) Collection from Côte d'Ivoire
}

\author{
Léonie Clémence Kouonon ${ }^{1 *}$, Kouamé Guillaume Koffi ${ }^{1}$, Koffi Adjoumani2 \\ Koudougnon Alice Estère Goba ${ }^{1}$ \\ ${ }^{1}$ Université Nangui Abrogoua, Unité de Phytotechnie et Amélioration Génétique, Abidjan, Côte d’Ivoire \\ ${ }^{2}$ Ecole Normale Supérieure d'Abidjan, Abidjan, Côte d'Ivoire \\ Email: *lckouonon@yahoo.fr, koffiguillaume2005@yahoo.fr, adjoumanidadon@gmail.com, aegoba@yahoo.fr
}

How to cite this paper: Kouonon, L.C., Koffi, K.G., Adjoumani, K. and Goba, K.A.E. (2020) Morphological Variability of Neglected and Underutilized Legumes (NULs) Collection from Côte d'Ivoire. American Journal of Plant Sciences, 11, 226-244. https://doi.org/10.4236/ajps.2020.112018

Received: December 3, 2019

Accepted: February 18, 2020

Published: February 21, 2020

Copyright $\odot 2020$ by author(s) and Scientific Research Publishing Inc. This work is licensed under the Creative Commons Attribution International License (CC BY 4.0).

http://creativecommons.org/licenses/by/4.0/ (c) (i) Open Access

\begin{abstract}
In order to characterize neglected and underutilized legumes seeds (NULs), prospection, collection and characterization were carried out in five administrative regions from Côte d'Ivoire. Two thousand and fifty-four accessions were collected. NULs cultivation is done mainly by women ( $88.1 \%$ compared to $11.9 \%$ of men). Productors distinguish 22 morphotypes of NULs based on seeds shapes, colors and patterns. Morphological traits of the collected morphotypes revealed a significant phenotypic polymorphism. Quantitative analyses showed a clear structure of accessions; three main groups were revealed. The first group contains accessions with large and thick seeds, but a low index shape opposite to group II with high index shape. Seeds of group III are characterized by small width and thickness, but a high shape index. Analyses of qualitative traits revealed five groups characterized by heterogeneous or homogeneous coloured integument, inked or elevated edge of hilum, presence or absence of eye.
\end{abstract}

\section{Keywords}

Neglected Legumes, Characterization, Quantitative Traits, Qualitative Traits

\section{Introduction}

Natural assets of Côte d'Ivoire (quality and availability of cultivable land, hydraulic system, favorable climate and varied vegetations) have favored the diversification of its agriculture, pillar of national economy. The success of this agricultural economy depends on the diversification of crop production, including 
cash or industrial crops (coffee, cocoa, rubber, pineapple, cotton, cashew nuts, etc.) and starchy food crops (sorghum, millet, beans, yams, manioc, taro, plantain bananas, corn, rice, etc.) [1] that guarantee financial and food security. In the margins of both categories, appear native but underutilized plant species. They belong to the traditional production category, specifically the sector of minor crops. Minor farms are characterized by low productivity, small size of cultivated areas, the hardness of work due to its manual nature, difficult access to agricultural inputs because their financing is scarce and an almost exclusively female workforce. At the heart of Côte d'Ivoire's neglected food heritage are many legumes whose dry seeds are used as a sauce ingredient.

In Côte d'Ivoire, they are generally referred to "beans" because they are characterized by a vinescent habit. Legumes are a part of the Fabaceae, generally annual, for food purposes and whose seeds are contained in pods. Groundnuts, cowpeas, voandzou, pigeon pea... are the most common pulses and are grown in larger areas [2]. Like these plants, little-known legumes, which are qualified as orphan and/or minor plants, play an important part in a sustainable diet. Many studies highlight their nutritional, agronomic, environmental and economic interest. Indeed, legume seeds are characterized by a high energy and nutritional content. Their high protein content ( $18 \%$ to $30 \%$, two to three times more than the seeds of major cereals), carbohydrates and minerals [3] [4] are the dietary assets available in the context of a healthy and balanced diet. In addition, their consumption pattern supports long storage without loss of nutritional value and adapt to droughts and marginal environments. Some of them contribute to improving the biological renewal of phosphorus. Moreover, thanks to their symbiotic nitrogen fixation capacity, they contribute to preserving soil fertility and improving the productivity of nutrient-poor sub-Saharan soils. Legumes reveal a wide variety of phenotypes. In fact, the color of the seed, the shape and the size of the pods are the units of diversity on which farmers classify local varieties. As a result of natural and human selection, these local varieties have accumulated genetic diversity that has been little studied and is therefore poorly exploited in the management of available plant genetic resources. In addition, due to changes in culinary practices, the cultivation of orphan legumes is rapidly declining in traditional production systems and yields are low and irregular. Only a few women farmers have kept the habit of cultivating them, but mainly on very small surfaces or in hut farming, just for domestic consumption needs. These changes in practices could lead to significant erosion of the genetic diversity of these legumes with consumable seeds. And insufficient genetic diversity increases risks for food security and nutrition. Knowledge of the genetic diversity of a resource is the pillar, the foundation of food security.

The rise of underutilized legumes therefore requires a better preservation of its genetic diversity. Very few studies of valorization of these species in Côte d'Ivoire have been carried out and do not enable to assess the level of genetic diversity of these minor plants. Indeed, the taxonomy is not updated, the collec- 
tion of genetic resources, the genetic characterization and the agronomic evaluation are fragmented or trivial. There is therefore an urgent need to prospect the country in order to assess the genetic variability and to constitute a germoplasm of these legumes for sustainable management of these resources.

\section{Methodology}

\subsection{Prospecting and Collection}

\subsubsection{Study Environment}

This study was carried out in Côte d'Ivoire, a country located in west Africa, between the meridians $2^{\circ} 30^{\prime}$ and $8^{\circ} 30^{\prime}$ west longitude and latitudes $4^{\circ} 30^{\prime}$ and $10^{\circ} 30^{\prime}$ north. Figure 1 points out the samples collection regions. The prospecting and collection areas include five administrative regions: District of Abidjan (south, regional capital, Abidjan), Sud-Comoé (south-east, regional capital, Aboisso), N'Zi (central, regional capital, Dimbokro), Moronou (central-east, regional capital, Bongouanou), Indénié-Djuablin (east, regional capital, Abengourou). These regions are included in Guinean agro-ecological zone. The climate is sub-equatorial type, characterized by two (02) rainy seasons and two (02) dry seasons. A long rainy season from April to July, during which $2 / 3$ of the annual

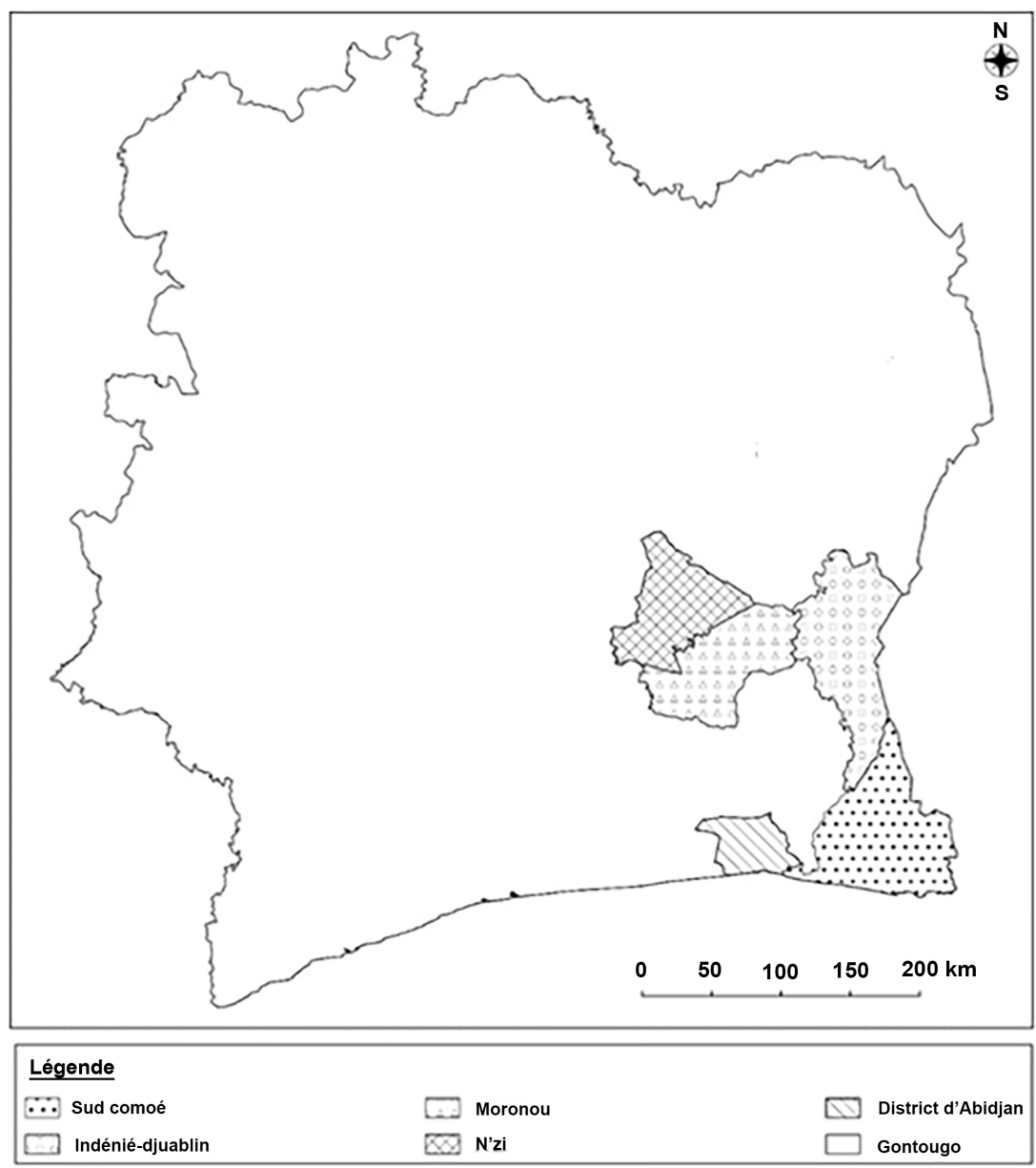

Figure 1. Administrative regions from Côte d'Ivoire of NULs sampled. 
rainfall falls, a minor dry season from August to September, a minor rainy season from October to November and a long dry season from December to March. The average rainfall is between 1300 and $1600 \mathrm{~mm}$ /year for 115 rainy days. Average temperatures range from $26^{\circ} \mathrm{C}$ to $28^{\circ} \mathrm{C}$ and can reach up to $30^{\circ} \mathrm{C}$ during the dry season.

\subsection{Data Collection}

\subsubsection{Sampling and Data Collection}

Prospecting and collection were conducted from December 2017 to January 2018 during the harvest season. Within each administrative region, sampling was stratified following departments, villages and fields.

\subsubsection{Ethnobotany}

Ethnobotanical data collection was conducted on 193 farmers randomly selected from 56 locations. Respondents were interviewed individually on the basis of a survey. The main data collected during the surveys relate to the gender of the people (women or men) practicing the crop, the ethnic group, the cultivation method, the identification and selection criteria for the morphotypes cultivated, cultivated areas, seed storage and acquisition methods.

\subsubsection{Identification of Morphotypes}

Samples of different morphotypes were submitted for identification to the Centre National de Floristique of Félix Houphouët-Boigny University, Abidjan, Côte d'Ivoire. The identification and classification of the different morphotypes seed observed in the entire collection was performed using a combination of several parameters: seed shape, color and integument pattern, shape and color of the eye pattern around the hilum.

\subsubsection{Characterization of Accessions}

The identification and classification of the different types of legumes collected during the survey missions were carried out. Morphological data were taken on all accessions collected, based on thirteen descriptors, including four quantitative and nine qualitative [5] [6]. These descriptors have been modified for the purposes of the study.

The 9 qualitative parameters studied present two to nine modalities. These parameters describe the seed coat (uniform or heterogeneous colouring, background colour, colour and appearance of the pattern if present), the hilum (appearance and perimeter) and the eye of the seed (presence or absence, colour, extent) (Table 1). Observations were made on 10 seeds per accession. On each seed, length, width and thickness were measured using a caliper (Mastercraft model; $15 \mathrm{~cm}$ long; accuracy $\pm 0.1 \mathrm{~mm}$ ) and the shape index estimated according to the ratio width to length of the seed.

\subsubsection{Processing of Prospecting, Collection and Survey Data}

The survey data were synthesized. The enumeration and inventory of accessions in each zone are translated into comparative tables. Abundance and distribution 
Table 1. Qualitative parameters used to describe the seeds of accessions collected from legumes.

\begin{tabular}{|c|c|c|c|}
\hline Traits & Traits codes & Modalities & Modalities Code \\
\hline \multirow{2}{*}{ Uniformity of colour } & \multirow{2}{*}{ UnCo } & Homogeneous colouring & UnCo1 \\
\hline & & Heterogeneous colouring & $\mathrm{UnCo} 2$ \\
\hline \multirow{9}{*}{ Background coloration } & \multirow{9}{*}{$\mathrm{CoFo}$} & White & $\mathrm{CoFo1}$ \\
\hline & & Cream & $\mathrm{CoFo} 2$ \\
\hline & & Grey & $\mathrm{CoF} 3$ \\
\hline & & Orange colour & $\mathrm{CoFo} 4$ \\
\hline & & Light brown & CoFo5 \\
\hline & & Purple & CoFo6 \\
\hline & & Red-violet & $\mathrm{CoFo} 7$ \\
\hline & & Burgundy red & $\mathrm{CoF} 88$ \\
\hline & & Black & $\mathrm{CoF} 09$ \\
\hline \multirow{6}{*}{ Pattern color } & \multirow{6}{*}{ CoMo } & Absent & CoMol \\
\hline & & Grey & $\mathrm{CoMo} 2$ \\
\hline & & Dark brown & CoMo3 \\
\hline & & Red & CoMo4 \\
\hline & & Purple & CoMo5 \\
\hline & & Black & CoMo6 \\
\hline \multirow{5}{*}{ Pattern aspect } & \multirow{5}{*}{ AsMo } & Absent & AsMo1 \\
\hline & & Speckled on the back of the seed & AsMo2 \\
\hline & & Speckled all over the surface of the seed & AsMo3 \\
\hline & & Marbled & AsMo4 \\
\hline & & Radial & AsMo5 \\
\hline \multirow{2}{*}{ Hilum periphery } & \multirow{2}{*}{$\mathrm{PoHi}$} & Flat & PoHil \\
\hline & & Raised & PoHi2 \\
\hline \multirow{2}{*}{ Eye pattern } & \multirow{2}{*}{ MoOe } & Absent & MoHil \\
\hline & & Present & $\mathrm{MoHi} 2$ \\
\hline \multirow{3}{*}{ Hilum appearance } & \multirow{3}{*}{ AsHi } & Pressed & AsHil \\
\hline & & Flat & AsHi2 \\
\hline & & Raised & AsHi3 \\
\hline \multirow{8}{*}{ Eye color } & \multirow{8}{*}{ CoOe } & Absent & CoHil \\
\hline & & White & $\mathrm{CoHi} 2$ \\
\hline & & Cream & $\mathrm{CoHi3}$ \\
\hline & & Grey & $\mathrm{CoHi} 4$ \\
\hline & & Dark brown & $\mathrm{CoHi5}$ \\
\hline & & Burgundy-red & CoHi6 \\
\hline & & Purple & $\mathrm{CoHi7}$ \\
\hline & & Black & $\mathrm{CoHi} 8$ \\
\hline \multirow{4}{*}{ Eye size } & \multirow{4}{*}{ EtOe } & Absent & EtOe1 \\
\hline & & Thin & EtOe2 \\
\hline & & Large & EtOe3 \\
\hline & & Very large & EtOei4 \\
\hline
\end{tabular}


of different seed morphotypes in all accessions were analyzed following proportions and histograms were performed.

\subsection{Statistical Analysis}

Descriptive and exploratory statistics were performed, on the one hand, to describe the total population, and on the other hand to estimate the differentiation level among accessions. For each morphotype and the whole population, the frequencies of qualitative modalities and the mean and the standard deviation of the quantitative parameters were calculated. The difference among morphotypes was tested by analysis of variance (ANOVA) for quantitative variables. Correlation among different parameters was tested by the Pearson correlation coefficient. Principal Component Analysis (PCA) was performed on non-correlated reduced-center variables.

The multiple correspondence analysis of qualitative morphological traits coded as ordinal data was used to identify all the morphotypes studied and determine the morphological parameters that contributed to differentiation among morphotypes.

\section{Results}

\subsection{Result of the Investigation}

\subsubsection{Socio-Demographic Profile of Producers}

One hundred and ninety-three producers were interviewed during our study. The vast majority of these respondents are female (Figure 2), 88.1\% compared to $11.9 \%$ of 19 to 78 -year-old men. The largest proportion of age groups is 40 49 , the lowest is under 19-year-old (Figure 3). In all the regions surveyed, culture is mainly practiced by native people (97.5\%, Figure 4). None of the 193 interviewees grow pure culture of legume. Neglected legumes are sown in associated culture with maize, cassava, groundnuts, yams or any other food crop. In all surveyed areas, cultivation is done behind huts or in the fields, at a rate of 1 to 5 plants in a field. The seed is acquired either by inheritance $(77 \%)$ or by donation (23\%). None of 193 people mentioned buying their seeds.

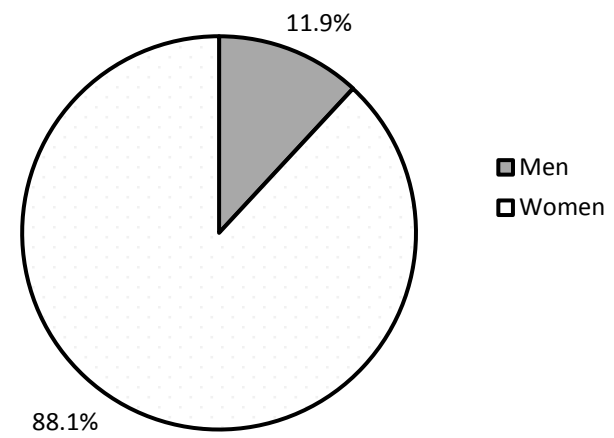

Figure 2. Proportions of farmers cultivating NULs in five administrative regions from Côte d'Ivoire based on gender. 


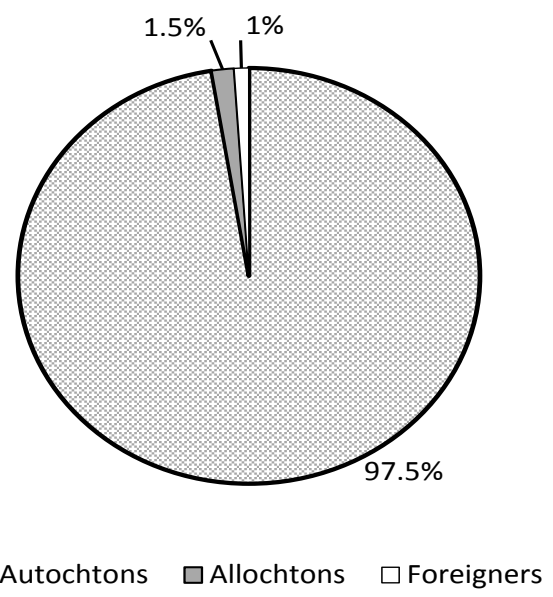

Figure 3. Proportions, based on origin, of farmers cultivating NULs in five administrative regions from Côte d'Ivoire.

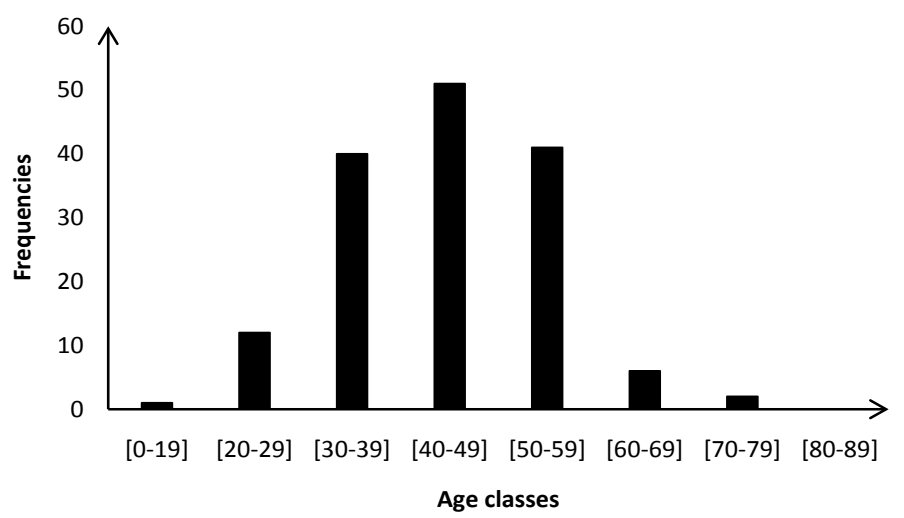

Figure 4. Histogram of age classes of farmers cultivating NULs.

\subsubsection{Uses}

From the surveys carried out, it appears that minor seed legumes are mainly grown for human consumption. According to producers, seeds do not have the same taste qualities and are used in the preparation of several traditional dishes where they have several uses. Peeling, fresh or dry, then removing their integument, they can be reduced to a paste to be used as an ingredient for sauces. They can also be simply added to the various dishes being cooked and thus replace meat or fish. They can still be the main dish and can be prepared in stew or sauce. Children are fond of these fresh or dry seeds that are simply cooked in water.

\subsubsection{Farmers' Diversity Management}

Respondents distinguish 22 morphotypes (Figure 5) of neglected legumes on the basis of shapes and colours and their patterns.

\subsubsection{Physical Characteristics of Accessions and Identified Seed Diversity}

Prospecting and collection missions enable to gather 254 accessions of orphan legume seeds. 


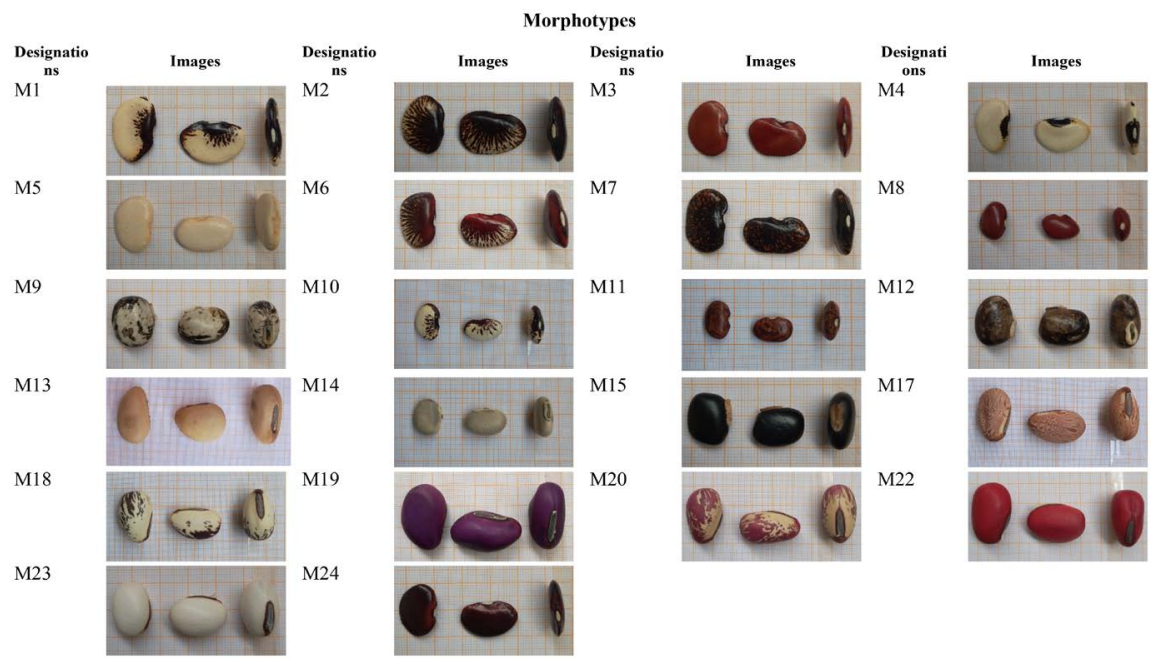

Figure 5. Classifications following seed size and colour of the 22 morphotypes of seed legume collected.

The 22 morphotypes identified by the farmers and collected in 47 of the 56 villages surveyed were carefully examined. Taxonomic identification allowed to classify them into four species Phaseolus lunatus, Canavalia ensiformis, C. gladiata, Mucuna pruriens and one indefinite species Phaseolus.

Figure 6 shows the numerical superiority of accessions of the species Phaseolus lunatus in all surveyed regions. Indénié-Djuablin region hold concurrently the five species of the legume's seeds identified. The cultivation of the identified minor legumes seed is not uniform across all surveyed areas. It goes from 5 morphotypes used in the Sud-Comoé region to 13 in the Indénié-Djuablin region (Figure 7).

\subsection{Morphological Description}

Results of the morphological description of the accessions of minor consumable legumes seeds from Côte d'Ivoire are summarized in Table 2. The ANOVA performed on the basis of the quantitative traits seed length, seed width, seed thickness and seed shape index show a very significant morphotype effect for each of the four quantitative parameters (Table 2). However, they only allow a partial distinction among morphotypes. Results of the LSD test indicate that three of these parameters allow the identification of 6 seed categories while only one of these four traits reveals only three groups.

Length of the seed allows to distinguish six groups: group 1 (M13, M19, M20, and M22); group 2 (M17, M18, M19, M20 and M22); group 3 (M1, M2, M3, M4, M5, M6, M7, M23 and M24), group 4 (M10 and M24), group 5 (M9, M10, M12, M14 and M15), group 6 (M8, M9, M11, M14 and M15).

The width of the seed highlights six groups: Group 1 (M13, M19, M20 and M22), group (M19, M20, M17, M18, M22 and M23), group 3 (M3, M4, M17 and M23), group 4 (M1, M2, M3, M4, M5, M6, M7, M9, M12, M14, M15, M23 and M24), group 5 (M9, M10, M11, M14 and M15) and group 6 (M8, M9, M10, M10, M11 and M14). 


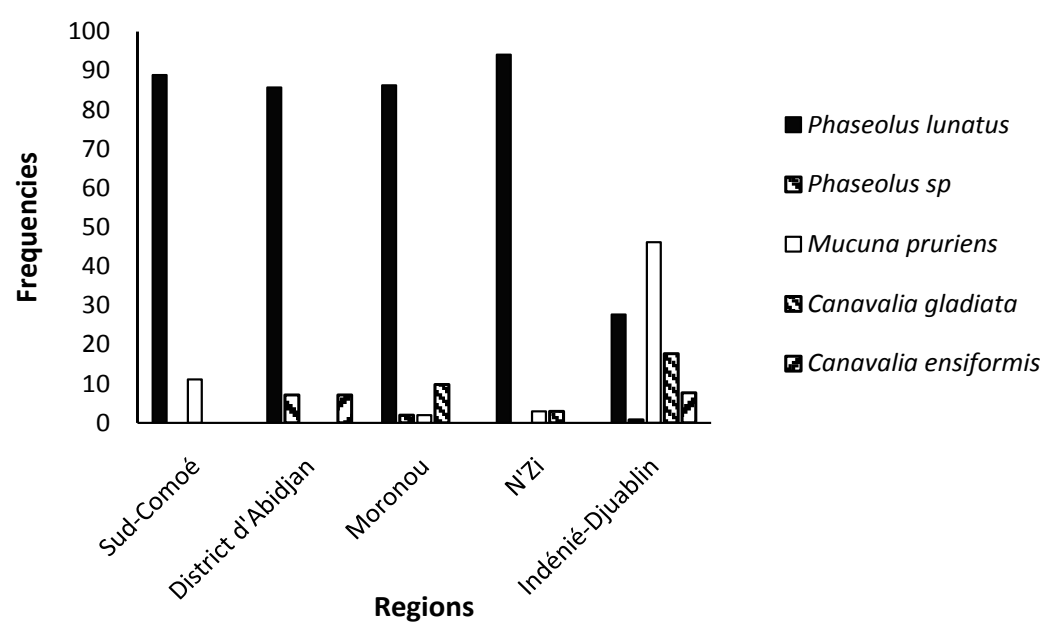

Figure 6. Distribution of five neglected legume species identified in five administrative regions of Côte d'Ivoire.

The six groups revealed by seed thickness are: group 1 (M13 and M22), group 2 (M17, M19, M20 and M22), group 3 (M17, M18, M19, M20 and M23), group 4 (M9 and M12), group 5 (M15 and M14), and group 6 (M1, M2, M3, M4, M5, M6, M7, M8, M10, M11, M14 and M24). The shape index made it possible to form three groups: group (M9, M11, M12, M12, M14, M14, M15 and M23), group 2 (M8, M11, M12, M14, M15, M22 and M23) and group 3 (M1, M2, M3, M4, M5, M6, M7, M8, M10, M11, M13 M14, M17, M18, M20, M22, M23 and M24). The correlation matrix (matrix not presented in this manuscript) shows positive and significant values $(\geq 0.50)$ in one pair of variables (LoGr-LaGr). Therefore, LoGr was eliminated in the multivariate analysis to avoid redundancies.

The first two axes of the AFD whose own value is greater than 1 were selected to describe total variability. They contain $96.96 \%$ of the variability among accessions (Table 3). The first axis is strongly correlated to the width and thickness of the seed. Axis 2 was significantly correlated with the width of the seed. Projections of variables (Figure 8) and individuals (Figure 9) in the factorial plane of the first two axes were used to analyze the morphological variability of legume seed morphotypes. The point cloud (Figure 9) shows a clear structure of accessions. It thus highlights three main groups in relation to axis 1 . The first set (I), located on the positive side, contains accessions of large and thick seeds but a low index shape, contrary to those located on the negative side (set II). Accessions of groups III are characterized by seeds of small width and thickness, but a high shape index. In order to determine the nature and degree of divergence and similarity between the 22 morphotypes studied, an Ascending Hierarchical Classification (AHC) was performed on the data of the quantitative parameters evaluated (Figure 10).

\subsection{Polymorphism of the Qualitative Morphological Characteristics Studied}

Analyses of the qualitative traits of the seeds of the collected accessions revealed 
Table 2. Mean values ( \pm standard deviation) of the four quantitative parameters measured in 22 morphotypes of seed legumes collected in eastern Côte d'Ivoire.

\begin{tabular}{|c|c|c|c|c|c|}
\hline Species & Morphotypes* & Length & Width & Thickness & Index shape \\
\hline \multirow[t]{9}{*}{ P. lunatus } & M01 (13) & $20.66 \pm 0.05^{c}$ & $13.36 \pm 0.076^{\mathrm{d}}$ & $5.32 \pm 0.04^{\mathrm{f}}$ & $0.65 \pm 0.03^{c}$ \\
\hline & M02 (10) & $21.26 \pm 0.05^{\mathrm{c}}$ & $13.67 \pm 0.07^{\mathrm{d}}$ & $5.94 \pm 0.04^{\mathrm{f}}$ & $0.64 \pm 0.03^{\mathrm{c}}$ \\
\hline & M03 (9) & $22.07 \pm 0.05^{c}$ & $14.18 \pm 0.07^{\mathrm{cd}}$ & $5.58 \pm 0.04^{\mathrm{f}}$ & $0.64 \pm 0.03^{c}$ \\
\hline & M04 (7) & $21.56 \pm 0.04^{\mathrm{c}}$ & $14.18 \pm 0.06^{\mathrm{cd}}$ & $5.41 \pm 0.03^{f}$ & $0.66 \pm 0.03^{c}$ \\
\hline & M05 (42) & $20.60 \pm 0.08^{c}$ & $13.56 \pm 0.11^{\mathrm{d}}$ & $5.34 \pm 0.06^{\mathrm{f}}$ & $0.66 \pm 0.03^{c}$ \\
\hline & M06 (10) & $21.09 \pm 0.05^{c}$ & $12.87 \pm 0.07^{\mathrm{d}}$ & $6.00 \pm 0.04^{\mathrm{f}}$ & $0.61 \pm 0.03^{c}$ \\
\hline & M07 (47) & $20.93 \pm 0.08^{c}$ & $13.72 \pm 0.12 \mathrm{~d}$ & $5.75 \pm 0.07^{\mathrm{f}}$ & $0.66 \pm 0.03^{c}$ \\
\hline & M10 (4) & $17.80 \pm 0.04^{\mathrm{de}}$ & $10.26 \pm 0.05^{\mathrm{ef}}$ & $5.79 \pm 0.03^{\mathrm{f}}$ & $0.58 \pm 0.03^{\mathrm{c}}$ \\
\hline & M24 (4) & $20.50 \pm 0.03^{\mathrm{cd}}$ & $12.72 \pm 0.04^{\mathrm{d}}$ & $5.44 \pm 0.03^{\mathrm{f}}$ & $0.62 \pm 0.04^{\mathrm{c}}$ \\
\hline Phaseolus & M08 (4) & $13.63 \pm 0.04^{\mathrm{f}}$ & $9.04 \pm 0.05^{\mathrm{f}}$ & $4.92 \pm 0.03^{f}$ & $0.66 \pm 0.02^{\mathrm{bc}}$ \\
\hline$s p$. & M11 (2) & $13.5 \pm 0.03^{\mathrm{f}}$ & $9.11 \pm 0.05^{\mathrm{ef}}$ & $5.09 \pm 0.02^{f}$ & $0.67 \pm 0.04^{\mathrm{abc}}$ \\
\hline \multirow[t]{4}{*}{ M. pruriens } & M09 (10) & $16.27 \pm 0.05^{\mathrm{ef}}$ & $12.44 \pm 0.07^{\mathrm{de}}$ & $9.48 \pm 0.04^{\mathrm{d}}$ & $0.77 \pm 0.04^{\mathrm{a}}$ \\
\hline & M12 (39) & $17.41 \pm 0.08^{\mathrm{e}}$ & $12.61 \pm 0.11^{\mathrm{d}}$ & $8.79 \pm 0.06^{\mathrm{d}}$ & $0.73 \pm 0.04^{\mathrm{ab}}$ \\
\hline & M14 (2) & $15.76 \pm 0.03^{\mathrm{ef}}$ & $11.38 \pm 0.05^{\mathrm{def}}$ & $6.85 \pm 0.02^{\text {ef }}$ & $0.72 \pm 0.03^{\mathrm{abc}}$ \\
\hline & M15 (13) & $15.94 \pm 0.05^{\mathrm{ef}}$ & $11.57 \pm 0.08^{\mathrm{de}}$ & $7.85 \pm 0.04^{\mathrm{e}}$ & $0.73 \pm 0.04^{\mathrm{ab}}$ \\
\hline \multirow[t]{2}{*}{ C. ensiformis } & M13 (11) & $29.85 \pm 0.05^{\mathrm{a}}$ & $19.69 \pm 0.07^{\mathrm{a}}$ & $16.73 \pm 0.04^{\mathrm{a}}$ & $0.66 \pm 0.03^{c}$ \\
\hline & M23 (2) & $20.86 \pm 0.03^{c}$ & $14.37 \pm 0.05^{\mathrm{bcd}}$ & $12.67 \pm 0.02^{c}$ & $0.69 \pm 0.04^{\mathrm{abc}}$ \\
\hline \multirow[t]{7}{*}{ C. gladiata } & M17 (6) & $26.66 \pm 0.04^{\mathrm{b}}$ & $16.01 \pm 0.06^{b c}$ & $14.46 \pm 0.03^{b c}$ & $0.61 \pm 0.03^{c}$ \\
\hline & M18 (12) & $25.77 \pm 0.05^{\mathrm{b}}$ & $16.43 \pm 0.07^{\mathrm{b}}$ & $13.78 \pm 0.04^{c}$ & $0.64 \pm 0.03^{c}$ \\
\hline & M19 (4) & $28.26 \pm 0.03^{\mathrm{ab}}$ & $17.62 \pm 0.05^{\mathrm{ab}}$ & $14.75 \pm 0.03^{b c}$ & $0.62 \pm 0.03^{c}$ \\
\hline & M20 (2) & $28.83 \pm 0.03^{\mathrm{ab}}$ & $17.39 \pm 0.05^{\mathrm{ab}}$ & $14.75 \pm 0.02^{\mathrm{bc}}$ & $0.60 \pm 0.04^{c}$ \\
\hline & M22 (2) & $27.67 \pm 0.03^{\mathrm{ab}}$ & $18.32 \pm 0.05^{\mathrm{ab}}$ & $16.47 \pm 0.02^{\mathrm{ab}}$ & $0.66 \pm 0.04^{\mathrm{bc}}$ \\
\hline & F & 23.9138 & 9.1058 & 70.4659 & 2.8352 \\
\hline & $P$ & $<0.0001$ & $<0.0001$ & $<0.0001$ & $<0.0001$ \\
\hline
\end{tabular}

Number in brackets in the "morphotypes" column indicates the number of accessions collected by morphotypes. The letters expounding on the standard deviation means indicate the homogeneous groups of morphotypes according to the least significant difference.

Table 3. Eigenvalues, percentage of discrimination in axes 1 and 2 and correlation coefficients between variables and axes.

\begin{tabular}{ccc}
\hline Axes & Axis 1 & Axis 2 \\
\hline Eigenvalues & 7.2842 & 3.1491 \\
Discrimination (\%) & 67.6932 & 29.2647 \\
Cumulated percentage (\%) & 67.6932 & 96.9579 \\
Seed width & $\mathbf{0 . 5 0 0 3}$ & $\mathbf{0 . 5 2 9 3}$ \\
Seed thickness & $\mathbf{0 . 9 3 6 8}$ & 0.1963 \\
Index shape & -0.0550 & -0.4814 \\
\hline
\end{tabular}




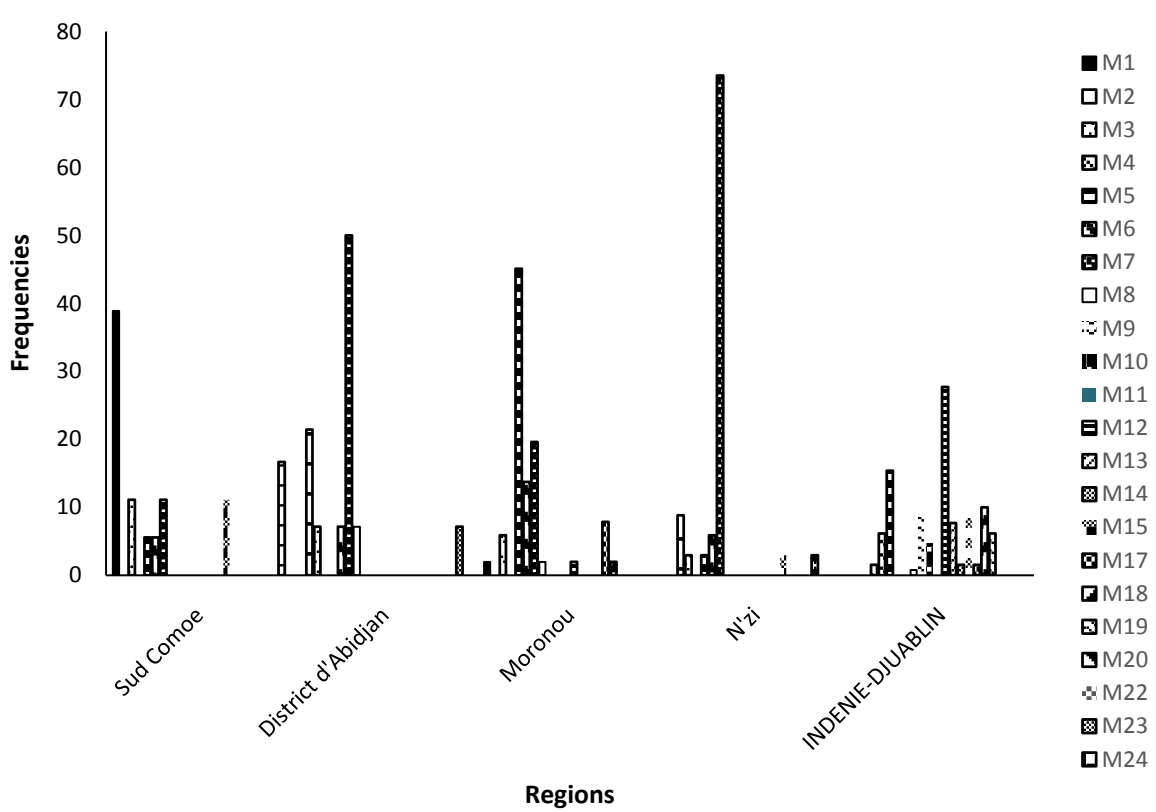

Figure 7. Distribution of the 22 morphotypes of neglected legumes identified in the five administrative regions of Côte d'Ivoire.

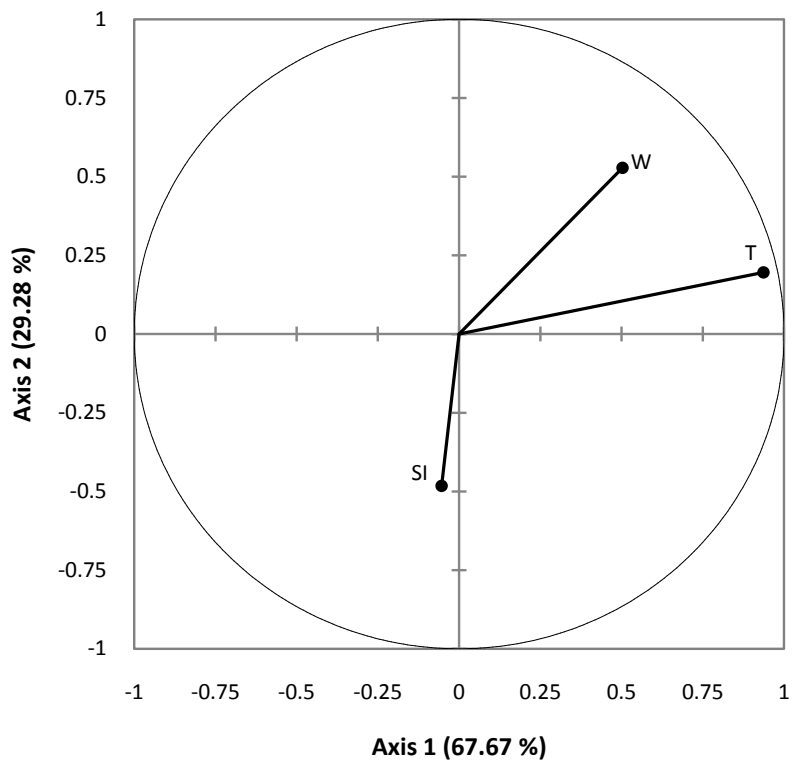

Figure 8. Circle of correlations of discriminant variables [width (W); thickness (T); shape index (SI)] the factorial plane of axes 1 and 2 .

a significant phenotypic polymorphism. Among the 41 categories of the nine descriptors used, 29 categories are present in all the morphotypes collected. Ten categories can be considered as rare categories, they are observed only in a single morphotype of minor seeded legumes among the 22 studied. These are the background colour categories light brown and purple respectively (morphotypes M11, M19 and M22 respectively). The colour of the pattern is also considered rare for seeds with heterogeneous integument, dark brown (M11) and purple 


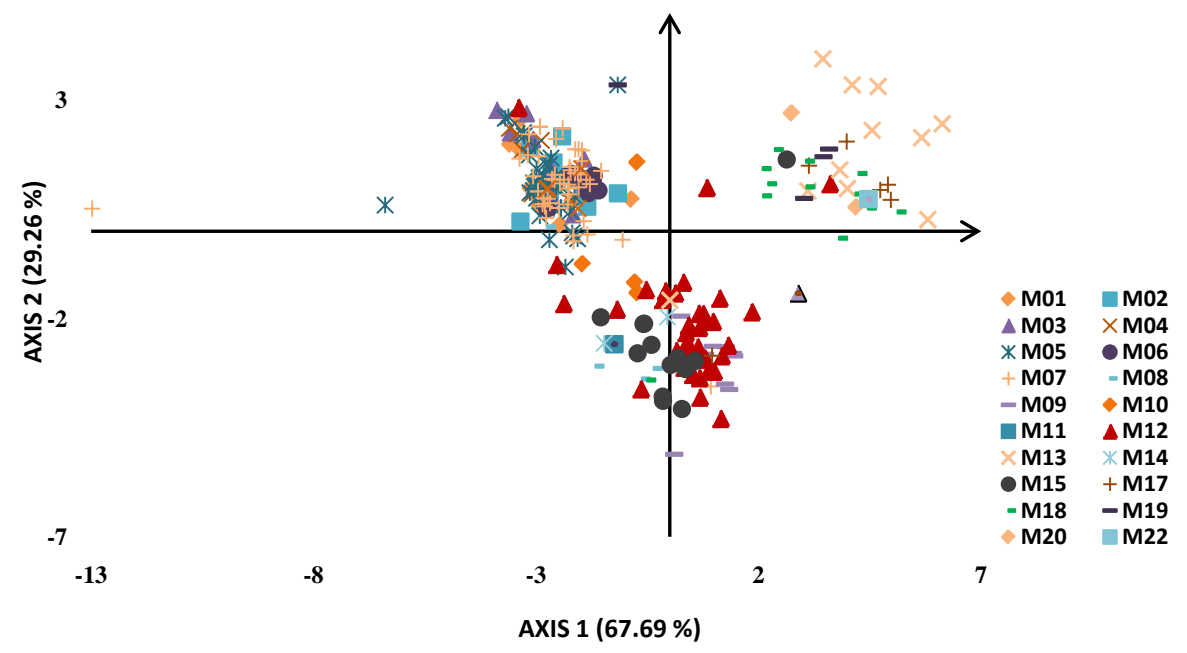

Figure 9. Ordination of morphotypes in the factorial plane of axes 1 and 2 of a discriminating factorial analysis.

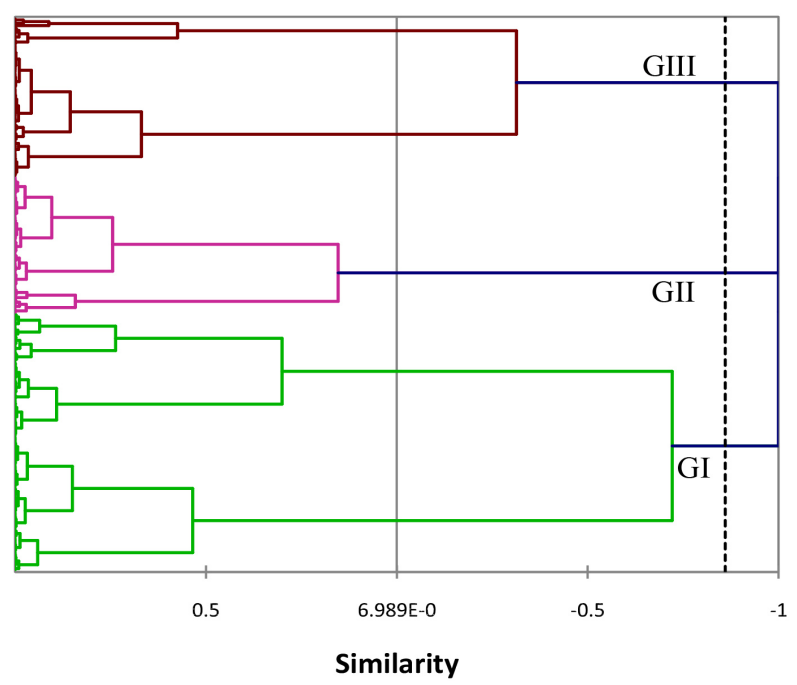

Figure 10. Dendrogram of the agglomerative hierarchical classification of 254 accessions based on discriminating characteristics in three groups (GI, GII and GIII).

(M20); the colour categories of the pattern around the hilum are cream (M9 and M14, 12 individuals), dark brown (M11, 2 accessions), burgundy red (M17, 6 accessions) and purple (M20, 2 accessions) and the very broad eye around the hilum (M2, 10 individuals).

The Multiple Correspondence Analysis (MCA), which enable to determine for all nine qualitative characteristics those that contribute most to the identification of the 254 individuals studied (which best explain the variability within the collection), shows that the phenotypic characteristics have been grouped according to the first two factorial axes. These axes accounted for $66.16 \%$ of the total adjusted inertia expressing the variability between cultivars within accessions (Table 4, Table 5). The inertia explained by this first two axis being $42.77 \%$ and $23.38 \%$ respectively. Moreover, based on the contributions of qualitative traits 
Table 4. Morphotype groups according to the hierarchical ascending classification.

\begin{tabular}{cl}
\hline Groups & \multicolumn{1}{c}{ Morphotypes (accession number) } \\
\hline \multirow{3}{*}{ GI } & M2 (7); M4 (4); M5 (19); M6 (9); M7 (29); M10 (1); M12 (3); M13 (10); M15 \\
& (1); M17 (5); M18 (11); M19 (3); M20 (2); M22 (2); M24 (2) \\
GII & M1 (9); M2 (3); M3 (3); M4 (3); M5 (21); M6 (1); M7 (15); M8 (1); M12 (2); \\
& M14 (1º); M24 (2) \\
GIII & M7 (3); M8 (3); M9 (10); M10 (3); M11 (2); M12 (33); M13 (1); M14 (1); \\
& M15 (12); M17 (1);M18 (1);M23 (2). \\
\hline
\end{tabular}

Table 5. Eigenvalues and percentages of inertia for the first two axes 1 and 2.

\begin{tabular}{ccc}
\hline Axes & Axis 1 & Axis 2 \\
\hline Eigenvalues & 0.660 & 0.517 \\
Ajusted inertia & 0.381 & 0.209 \\
Ajusted inertia (\%) & 42.772 & 23.388 \\
Cumulated percentage & 42.772 & 66.160 \\
\hline
\end{tabular}

(7\%) to the formation of these selected axes, only 12 of the 41 modalities observed explain the morphological variations within the collection. The 12 modalities come from 8 of the 9 traits examined (Table 6). The other characteristics are not very representative in the factorial plane of axes 1 and 2 and square cosines less than 0.50 , and therefore their projection does not provide any useful information. These contributions indicate a higher identification potential for these parameters. Thus, axis 1 which describes $42.77 \%$ of the variation, is defined by 6 variables: uniformity of the color of the integument, color of the pattern on the integument, the appearance of this pattern, color and pattern of the eye, and extent of the eye. Axis 2, which expresses $23.39 \%$ of the total variability, is defined by the uniformity of the colouring, the appearance of the integument pattern, the circumference of the hilum, the appearance of the eye and its extent. Figure 11 shows five groups in the plane of the first two main components (Axis 1 and Axis 2). Axis 1 distinguishes between individuals in groups 1, 2, 3, 4 and 5, located respectively on the positive and negative side of the axis. Individuals of morphotypes M12, M09 and M14 forming group 1 belong to the species Mucuna pruriens. They are characterized by the edge of the raised hilum ( $\mathrm{PoHi} 2$ ), the protruding hilum (AsHi 3).

Individuals of morphotypes M01, M02, M06, M7, M10, M24 belonging to the species $P$. lunatus form group $\mathrm{V}$ occupy the negative part of axis 2 , which corresponds to individuals with a heterogeneous coloured integument, an inked hilum (AsHi2) and a broad eye (EtHi 3). On the positive side of axis 1 are groups 2 and 3. Group 2 consists of individuals of morphotype 15 only, characterized by a homogeneous colored integument (UnCo-1), the edge of the elevated hilum (PoHi 2), the protruding hilum (AsHi 3). Like group 3, it includes individuals of morphotypes M3, M4, M4, M5, M8, M13, M19, M22 and M23. There are therefore individuals of the species C. gladiata (M19, M22), C. ensiformis (M13, 
Table 6. Contribution of variables to the formation of the first two factorial axes.

\begin{tabular}{lcc}
\hline Axes & Categories & Contribution of categories (\%) \\
\hline Axis 1 & UnCo-1 & 8.2 \\
& CoMo-1 & 7.9 \\
& AsMo-1 & 7.9 \\
MoHi-2 & 9.5 \\
CoHi-1 & 9.6 \\
Axis 2 & EtHi-1 & 9.6 \\
& Cofo-4 & 7.5 \\
& PoHi-2 & 11.7 \\
& AsHi-1 & 8.5 \\
& AsHi-3 & 10.1 \\
& EtHi-3 & 9.9 \\
\hline
\end{tabular}

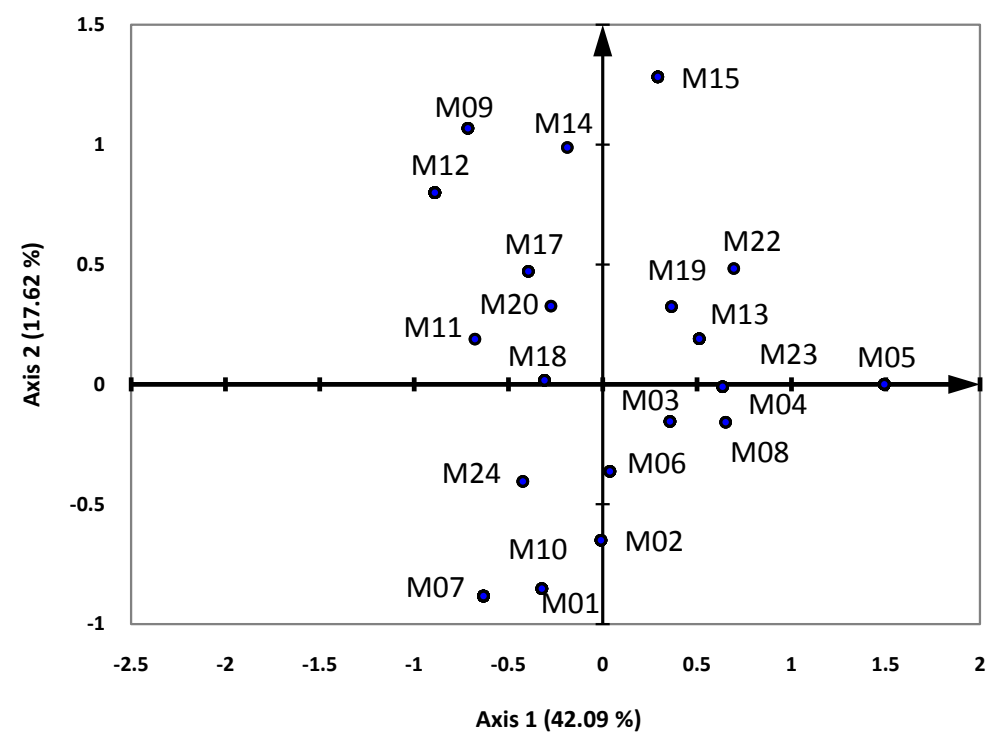

Figure 11. Multiple Correspondence Analysis (MCA) on all morphotypes based on qualitative traits.

M23), P. lunatus. (M3, M4 and M5) Phaseolus sp (M8) characterized by a homogeneous coloured integument with or without marked eye.

Group 5 formed by individuals of the species C. gladiata (M17, M18, M20) and an undetermined morphotype of Phaseolus (M11). Individuals in this group are represented by accessions characterized by a heterogeneously colored integument with marked speckling.

\section{Discussion}

This study was carried out in the context of the conservation of under-exploited plants genetic resources in Côte d'Ivoire. This is the first report characterizing the morphotypes of minor and endangered legumes seeds collected in Côte 
d'Ivoire. It enabled to assess the current state of the variability of these resources in their main production areas in Côte d'Ivoire, an essential step in assessing the diversity and genetic improvement of the resource [7] [8]. It makes it possible to provide plants genetic resources improvers and managers with vital information necessary for their work [7].

The data set obtained after a survey enabled to assess the variability within the "neglected legumes" resource. Farmers' management of this resource in its production area in Côte d'Ivoire has revealed 22 morphotypes of consumable legumes seeds. These morphotypes were identified by the Centre National de Floristique, which grouped them into five species: Phaseolus lunatus, Phaseolus sp., Mucuna pruriens, Canavalia ensiformis and C. gladiata. A collection of 254 accessions was selected to represent the diversity of morphotypes collected from 47 localities and five Ivorian administrative regions.

The collected accessions were characterized using morphological descriptors. Results of seeds quantitative traits analysis, namely seed length, seed width and thickness, and qualitative traits showed significant variability among morphotypes. Morphotypes are defined on the basis of seeds colour, shape and size. The cultivation of the identified minor legumes seeds is not uniformly distributed over the five surveyed regions. Also, species show morphological variability. $P$. lunatus is represented by nine morphotypes for 146 accessions, Phaseolus sp includes two morphotypes for six accessions, $M$. pruriens is represented by four morphotypes for 64 accessions, $C$. ensiformis contains two morphotypes for 15 accessions, $C$. ensiformis large seeds includes 1 morphotype for 13 accessions, $C$. ensiformis small seeds is represented by 1 morphotype for 23 accessions and $C$. gladiata includes 5 morphotypes for 26 accessions.

While $P$. lunatus is the most representative of collected species, it is present in varying proportions in each of the surveyed regions, idem for morphotypes. Indeed, morphotype M3 with only ( 9 accessions) is present in the five surveyed regions while morphotype M7 represented by 47 accessions is present in only four of the five surveyed regions. Some regions have a greater number of morphotypes than others, this is the example of Indénié-Djuablin region, which has collected the largest number of species and morphotypes (130 accessions for the five species of minor seeded legumes used in sauces, some are the main food or consumed as a treat or snack. The Moronou region is the only one where accessions of Canavalia ensiformis with large seeds, $C$. gladiata and the morphotypes M9 and M14 of $M$. pruriens have been collected. The greater variability of morphotypes from one region to another could also be explained by different social factors. Legumes are deeply integrated into the socio-cultural life and diet of local populations, and their large number in the Indénié-Djuablin region could be due to trade with neighbouring Ghana, a country where seed legume cultivation is more sustained. This would contribute to increasing the genetic diversity of local types in the region [9].

The analysis of variance showed a significant difference between the seeds of the accessions on all the parameters analyzed. The Principal Component Analy- 
sis, hierarchical bottom-up classification and discriminant factor analysis confirmed this important morphological variability. A strong phenotypic structuring of the studied material into three sets is highlighted. The first group includes 117 accessions characterized by large and thick seeds but a low index shape. The second group includes 62 accessions characterized by seeds of small width and thickness, but a high shape index, the $3^{\text {rd }}$ group includes 75 accessions.

However, ANOVA and the LSD test indicate that all traits vary from one morphotype to another, suggesting that legumes studied have been ecologically adapted [10]. The ACM has shown that the cumulative portion of information provided on the first two axis is $66.16 \%$, which is relatively high, allowing a better representation of the relationship among all the variables. This analysis confirms the variability of morphotypes within legume seeds groups. The study of seed variability for variety identification is common [11] [12] among Fabaceae. Parameters such as integument staining, eye and hilum appearance and staining are widely documented [13] [14], indicating the importance of seed characteristics in the study of systematics in the Fabaceae. [15] [16] [17] have shown that the variability of patterns on the seed surface is very useful for species recognition in the Fabaceae. Many differences in colouring, appearance within the same species, could be the result of an adaptive process. The great diversity that generally characterizes the Fabaceae indicates the presence of several mutations controlling morphological traits within the family itself. Ecologically, the Fabaceae family colonized all habitats (forests, deserts, plains to mountains). They are forage, ornamental, wild, domesticated, woody, as well as herbaceous... The emergence of variability in organisms can be explained by the fact that diversity ensures survival following evolution and sets the potential for an adaptive response to fluctuations in environmental conditions. Thus, the morphological differences observed among different morphotypes within a species or between species suggest that morphotypes are created and maintained under very different evolutionary processes within the same agroecosystem. At the population level, evolutionary processes can indeed lead to variations in morphological characteristics. And evolutionary processes could include anthropogenic factors. Indeed, anthropogenic factors are likely to exert highly variable selective pressures on genotypes [18]. They tend to modify genetic diversity. For example, the mode of seeds management influences morphotypes diversity. Morphotype choices and selections, as well as cultural practices, lead to a diversified selection resulting in the acquisition or maintenance of significant phenotypic diversity [19]. This morphological differentiation observed may be compromised by the gradual disappearance of species due to changes in farming practices. Many of the practices identified could ensure that diversity is maintained. Indeed, the acquisition of seeds by inheritance or donation, the poly-varietal culture, the eating habits of certain peoples, the culture mainly ensured by indigenous people, those who have kept in mind the ancestral eating habits of minor seed legumes are factors that can perpetuate the conservation of the resource's diversity. The age of producers indicates that adult and senior producers have more 
expertise in growing, consuming and producing pulses than younger generations. The lack of interest of younger generations in minor seed legumes in favour of crops with improved varieties hinders their maintenance and promotes their disappearance. According to interviewees, production appears to have declined over the years, due to the popularity of other legumes such as cowpea, groundnuts and soybeans. This study was also carried out with the objective of looking for the morphological characteristics that best explain the variety of diversity observed among producers among legumes seeds identified in Côte d'Ivoire. The identification of the peasant diversity management unit as an analytical unit can be an important tool for diversity assessment [20] [21]. The use of seed colour as a unit of diversity within the legume collection is an important differentiation criterion in farming environments. The grouping of genotypes within each diversity group generally shows a structuring of morphological variability according to the colour of the genotype and seems to confirm the peasant classification.

\section{Conclusion}

It is undeniable that several local varieties of neglected and underutilized legumes are available in the hands of farmers. In Côte d'Ivoire, producers in five resource-producing regions recognize 22 morphotypes. Certain varieties have their own qualitative and quantitative characteristics. Our results have highlighted the heterogeneity of the collection legumes collected. Significant differences among accessions, relating to the length, width, thickness and shape index of these morphotypes have been revealed by ANOVA. It was also possible in terms of quantitative traits (width, thickness and shape of the seed) and qualitative features (uniformity of coloring, patterns on the seed coat, appearance of the hilum, coloring and the appearance of the eye) to reveal groups of different sizes and morphologies within our germoplasm, thanks to multivariate analyzes (FDA, AHC, ACM).

\section{Conflicts of Interest}

The authors declare no conflicts of interest regarding the publication of this paper.

\section{References}

[1] Sangare, A., Koffi, E. and Akamou, F. (2009) Etat des ressources phylogénétiques pour l'alimentation et l'agriculture: Second rapport national. $65 \mathrm{p}$.

[2] Snapp, S., Rahmanian, M. and Batello, C. (2018) Légumes secs et exploitations durables en Afrique subsaharienne, sous la direction de T Calles. FAO, Rome, 69 p.

[3] Baudoin, J.P. (2001) Contribution des ressources phytogénétiques à la sélection variétale de légumineuses alimentaires tropicales. Biotechnologie. Agronomie, Society and Environnement, 5, 221-230.

[4] Rémond, D. and Walrand, S. (2017) Les graines de légumineuses: Caractéristiques nutritionnelles et effets sur la santé. Innovations Agronomiques, INRA, 60 p. 
[5] Woodger, T.A. (2011) The Pictorial Guide Seeds of the World: An Introduction into the Collection, Cleaning, and Storage. Universal Publisher, Boca Raton.

[6] Choudhary, N., Hamid, A., Singh, B., Khandy, I., Sofi, P.A., Bhat, M.A. and Mir, R.R. (2017) Insight into the Origin of Common Bean (Phaseolus vulgaris L.) Grown in the State of Jammu and Kashmir of Northwestern Himalayas. Genetic Resources and Crop Evolution, 65, 963-977. https://doi.org/10.1007/s10722-017-0588-Z

[7] Morillo, A.C., Tovar, Y.P. and Morillo, Y. (2016) Morphological Characterization of Selenicereus megalanthus (K. Schum. ex Vaupel) Moran in the Province of Lengupá. Ciencia en Desarrollo, 7, 2. https://doi.org/10.19053/01217488.v7.n2.2016.4072

[8] Sharma, A., Jain, D., Khandelwal, S.K., Chaudhary, R., Ameta, K.D. and Singh, A. (2019) Morphological, Biochemical, and Molecular Characterization of Orange-Fleshed Sweet Potato (Ipomoea batatas [L.] Lam) Germplasms. In: El-Esawi, M.A., Ed., Genetic Diversity in Plant Species: Characterization and Conservation, IntechOpen, London, 20. https://doi.org/10.5772/intechopen.82597

[9] Robert, T., Mariac, C., Allinne, C., Ali, K., Beidari, Y., Bezançon, G., Couturon, E., Moussa D., Sasou, M.S., Seydou, M., Seyni, O., Tidjani, M. and Luxereau, A. (2005) Gestion des semences et dynamiques des introgressions entre variétés cultivées et entre formes domestiques et spontanées des mils (Pennisetum glaucum ssp. glaucum) au Sud-Niger. Les Actes du BRG, 5e colloque national. Un dialogue pour la diversité. Lyon, 3-4-5 novembre 2004.

[10] Bonny, S.B. and Djè, Y. (2011) Variabilité morphologique et agronomique des variétés traditionnelles de voandzou [Vigna subterranea (L.) verdc. (Fabaceae)] de Côte d'Ivoire. Journal of Applied Biosciences, 41, 2820-2835.

[11] Najafabadi, S.S.M. and Farahani, L. (2012) Shape Analysis of Common Bean (Phaseolus vulgaris L.) Seeds Using Image Analysis. International Research Journal of Applied and Basic Sciences, 3, 1619-1623.

[12] Kara, M., Sayinci, B., Elkoca, E., Öztürk, I. and Özmen, T.B. (2013) Seed Size and Shape Analysis of Registered Common Bean (Phaseolus vulgaris L.) Cultivars in Turkey Using Digital Photography. Journal of Agricultural Sciences, 19, 219-234. https://doi.org/10.1501/Tarimbil 0000001247

[13] Turkic, Z., El-Shayeb, F. and Abozeid, A. (2014) Seed Morphology of Some Trigonella L. Species (Fabaceae) and Its Taxonomic Significance. International Journal of Science and Research, 3, 940-948.

[14] Gaafar, R.M., Hamouda, M. and Badr, A. (2016) Seed Coat Color, Weight and Eye Pattern Inheritance in Gamma-Rays Induced Cowpea M2-Mutant Line. Journal of Genetic Engineering and Biotechnology, 14, 61-68. https://doi.org/10.1016/j.jgeb.2015.12.005

[15] Fawzi, N.M. (2011) Macro- and Micro-morphological Seed Characteristics of Some Selected Species of Caesalpinioideae-Leguminosae. Research Journal of Botany, 6 , 68-77. https://doi.org/10.3923/rjb.2011.68.77

[16] Hussein, A.H., Ghareeb, A., Khalifa, S.F. and Fawzi, N.M. (2002) Taxonomic Importance of Seed Characteristics of Some Species of Cassia and Senna (Subfamily: Caesalpinioideae-Leguminosae). Egyptian Journal of Biotechnology, 12, 248-265.

[17] Hussein, A.H., Ghareeb, A., Khalifa, S.F. and Fawzi, N.M. (2002) Taxonomic Criteria of the Characteristic Features in Seeds of Some Selected Species of Caesalpinioideae-Leguminosae. Egyptian Journal of Biotechnology, 12, 280-298.

[18] Hendry, A.P., Gotanda, M.K. and Svensson, E.I. (2017) Human Influences on Evolution, and the Ecological and Societal Consequences. Philosophical Transaction of 
the Royal Society B, 372, Article ID: 20160028.

https://doi.org/10.1098/rstb.2016.0028

[19] Othmani, A., Mosbahi, M., Ayed, S., Slim-Amara, H. and Boubaker, M. (2015) Morphological Characterization of Some Tunisian Bread Wheat (Triticum aestivum L.) Accessions. Journal of New Sciences, Agriculture and Biotechnology, 15, 503-510.

[20] Agbolade, J.O., Olakunle, T.P., Popoola, K.M., Idowu, J.A., Aasa-Sadique, A.D. and Isiaka, A.I. (2019) Genetic Variability and Diversity Analysis in Pod and Seed Characters of Some Neglected and Underutilized Legumes (NULs). Asian Journal of Biochemistry, Genetics and Molecular Biology, 2, 1-8. https://doi.org/10.9734/ajbgmb/2019/v2i330059

[21] Gixhari, B., Vrapi, H. and Hobdari, V. (2014) Morphological Characterization of Pea (Pisum sativum L.) Genotypes Stored in Albanian Genebank. Albanian Journal of Agricultural Sciences, Special Edition, 169-173. 